\title{
A semi-discrete model and its approach to a solution for a wide moving jam in traffic flow
}

\author{
Peng Zhang ${ }^{1,3 *}$ Chun-Xiu $\mathrm{Wu}^{1,3}$ and S.C. Wong ${ }^{2}$ \\ 1. Shanghai Institute of Applied Mathematics and Mechanics, Shanghai University, \\ Shanghai, P.R. China \\ 2. Department of Civil Engineering, The University of Hong Kong, Pokfulam Road, \\ Hong Kong SAR, P.R. China \\ 3. Shanghai Key Laboratory of Mechanics in Energy Engineering
}

\begin{abstract}
This paper investigates the analytical and numerical solutions to wide moving jams in traffic flow. Under the framework of the Lagrange coordinates, a semi-discrete model and a continuum model correlate with each other, in which the former model approaches the latter as the increment $\Delta M$ in the former model vanishes. This implies that the solution to a wide moving jam in the latter model, which can be analytically derived using the known theory, can be conceivably taken as an approximation to that of the former model. These results were verified through numerical simulations. Because a detailed understanding of the traffic phase "wide moving jam" is very important for the further development of Kerner's three-phase traffic theory, this study helps to explain the empirical features of traffic breakdown and resulting congested traffic patterns that are observed in real traffic.
\end{abstract}

Keywords: Lagrange coordinates; semi-discrete model; car-following model; wide moving jam;

\section{Introduction}

Kerner $[1,2]$ introduced a "three-phase traffic theory" in which there are two phases in congested traffic: 1 . Synchronized flow and 2. Wide moving jam. This theory explains the traffic breakdown and other empirical features of traffic flow that were observed on different highway in various countries. In this three-phase theory, the traffic breakdown is described as a first-order phase transition from

\footnotetext{
*Corresponding author. E-mail: pzhang@mail.shu.edu.cn.
} 
free flow to synchronized flow $(\mathrm{F} \rightarrow \mathrm{S}$ transition), whereas wide moving jams are formed through a sequence of two phase transitions called $\mathrm{F} \rightarrow \mathrm{S} \rightarrow \mathrm{J}$ transitions: Firstly a $\mathrm{F} \rightarrow \mathrm{S}$ transition occurs and later and usually at another road location a $\mathrm{S} \rightarrow \mathrm{J}$ transition is realized.

A wide moving jam with $\mathrm{F} \rightarrow \mathrm{J}$ transition was first discovered by Kerner [3] based on a numerical and analytical study of a version of Payne's model [4]. Later, the results of [3] about wide moving jams have been incorporated and further developed in many traffic flow models, e.g., the characteristic features of wide moving jam phase in cellular automaton models $[5,6]$ and higher-order models [7-13]. Although most of these studies cannot show synchronized flow, thus unable to predict $\mathrm{F} \rightarrow \mathrm{S} \rightarrow \mathrm{J}$ transitions, they are important for the further development of traffic flow theory, because the characteristic features of wide moving jams play a very important role in Kerner's three-phase traffic theory. On the other hand, most semi-discrete (car-following) models (e.g., in [14-16]) failed to analytically show the characteristic features of a wide moving jam even through they are deterministic. This could be probably due to the unavailability of analytical solutions to most non-linear ordinary differential systems.

This paper studies the characteristic feature of wide moving jams in a semi-discrete model. Using the concept of the Lagrange coordinates, the formulated semi-discrete model with an increment $\Delta M$ could converge to a continuum higher-order model for $\Delta M \rightarrow 0$, where $\Delta M$ is the mass between two adjacent particles. This implies consistency between the semi-discrete and continuum models. Therefore, with the error of $O(\Delta M)$ the characteristic parameters of a wide moving jam in the former model can be well approximated by those in the latter model, which can be similarly derived through application of the weak solution theory of hyperbolic conservation laws, as was shown in Zhang et al. [11-13]. To verify the convergence, we numerically demonstrate that the semi-discrete model is able to reproduce a regular wide moving jam, and that for the refinement of $\Delta M$ its characteristic parameters do approach those that are analytically derived through the continuum model. Even with a large value of $\Delta M=1$, in which the semi-discrete model reduces to a car-following model, the approximation is also very good.

Here, we mention a similar establishment between the continuum and the car following models in Aw et al. [17] and Greenberg [8,18], in which the convergence of latter solution to the former solution was generally and indirectly showed by "shrinking" the time and space coordinates such that the length of a car approaches zero [17]. More relevantly, Greenberg's analytical study of traveling waves that was based on different assumptions should be suited more generally for a narrow and wide moving jam [8]. However, he did not show convergence and compare between analytical and numerical solutions. In this regard, the present paper also serves as a supplement to the aforementioned studies. We should also note that the formulation under the Lagrange coordinate system mostly results in 
"anisotropic" models, which are related to those discussed under the Euler coordinate system, e.g., in $[9,10,19-22]$.

The remainder of the paper is organized as follows. In Section 2, we discuss the basic concepts of one-dimensional fluid in relation to the Lagrange coordinates, based on which we formulate an acceleration equation for traffic flow. In Section 3, the linear stability condition of the equilibrium solution for the resulting continuum model is derived and an analytical wide moving jam solution is constructed. In Section 4, the semi-discrete model is naturally formulated based on the discussion in Section 2, and the aforementioned convergence of its solution for wide moving jam to that of the continuum model is demonstrated through numerical simulations. We conclude the paper in Section 5.

\section{General Discussion of Model Equations}

In most studies, the initial position of a particle is more generally taken as the Lagrange coordinates of the particle. However, for a one-dimensional continuum like traffic flow, the total mass upstream of a particle also remains unchangeable and thus is more conveniently used as the Lagrange coordinate to identify the particle. In this case, equations including the mass conservation and acceleration are easily established, and it is straightforward to derive the motion equations of particles by direct discretization of these equations.

\subsection{Lagrange Coordinates and Mass Conservation}

Let $M(x, t)$ denote the total mass not passing through position $x$ at time $t$ in a one-dimensional continuum. Then, the density of the fluid is defined as

$$
\rho(x, t)=\lim _{\Delta x \rightarrow 0} \frac{M(x+\Delta x, t)-M(x, t)}{\Delta x}=M_{x}(x, t),
$$

and the flow is defined as

$$
q(x, t)=-\lim _{\Delta t \rightarrow 0} \frac{M(x, t+\Delta t)-M(x, t)}{\Delta t}=-M_{t}(x, t) .
$$

Here, the mass $M$ measures the quantity of substance in the continuum, which is denoted as the number of cars in traffic flow. Application of the identity $M_{x t}=M_{t x}$ to Eqs. (1) and (2) in a smooth solution region gives rise to the mass conservation:

$$
\rho_{t}+q_{x}=0
$$

The formulation becomes the same as that in $[23,24]$ by replacing $M(x, t)$ with $-N(x, t)$, where $N(x, t)$ is the total mass passing through location $x$ at time $t$. 
Under the continuum hypothesis, the total mass not passing through a specific particle remains a constant in the motion or is independent of $x$ and $t$ because no overtaking is allowed. Moreover, for fixed $t$, Eq. (1) implies that $M$ is strictly increasing of $x$ as the vacuum is not considered or would be specially treated. Therefore, it is convenient to identify a specific particle in the motion by the index $M$ (instead of $x$ ) and time $t$, which constitutes the Lagrange coordinate system under Eqs. (1) and (2). Accordingly, we rewrite Eq. (1) as

$$
s(M, t)=x_{M}(M, t),
$$

where $s(M, t)=(\rho(x, t))^{-1}$ is called the specific volume at $(x, t)$ or $(M, t)$, and $x(M, t)$ is the position of particle $M$ at time $t$. The motion speed of particle $M$ at time $t$ is defined as

$$
u(M, t)=\lim _{\Delta t \rightarrow 0} \frac{x(M, t+\Delta t)-x(M, t)}{\Delta t}=x_{t}(M, t) .
$$

In this coordinate, the mass conservation in a smooth solution region can be similarly derived from the identity $x_{t M}=x_{M t}$, which leads to

$$
s_{t}-u_{M}=0
$$

Moreover, by the Lagrange coordinate transformation (1)-(2), the partial derivative $x_{t}(M, t)$ of the implicit function $x=x(M, t)$ can be derived from $M=M(x, t)$ as

$$
x_{t}(M, t)=-\frac{M_{t}(x, t)}{M_{x}(x, t)}=q(x, t) / \rho(x, t),
$$

which together with Eq. (4) gives the relation $q(x, t)=\rho(x, t) u(x, t)$. Here, the speed $u(x, t)$ of fluid at $(x, t)$ is defined as the motion speed $u(M, t)$ of particle $M(x, t)$.

\subsection{Acceleration in Fluid Dynamics}

The acceleration of particle $M$ at time $t$ is naturally defined as

$$
u_{t}(M, t)=\lim _{\Delta t \rightarrow 0} \frac{u(M, t+\Delta t)-u(M, t)}{\Delta t} .
$$

Recall that the speed of fluid at $(x, t)$ is defined as the speed of the corresponding particle $M$ at time $t$, i.e., $u(M, t)=u(x, t)$. Then, taking the derivative of this equation with respect to $t$ gives

$$
u_{t}(M, t)=u_{t}(x, t)+u_{x}(x, t) x_{t}(M, t)=u_{t}(x, t)+u(x, t) u_{x}(x, t),
$$

where the function $x=x(M, t)$ is implied. Therefore, we can further define the acceleration of fluid at $(x, t)$ as the acceleration of particle $M$ at time $t$, and the expression on the right-hand side is under the Euler $x$ - $t$ coordinate. 


\subsection{Acceleration of Traffic Flow}

Traffic flow acceleration is mainly associated with the anticipation and relaxation effects, and the acceleration under the Euler coordinate could be described according to the same or similar physical mechanisms as those in the literature (e.g., see [?,3,4,25]). However, it is also straightforward to formulate under the Lagrange coordinate system. We assume that the velocity of particle $M$ at time $t+\tau_{\epsilon}$ is determined by the velocity $u(M+\Delta M, t)$ of particle $M+\Delta M(\Delta M>0)$ and an equilibrium velocity $u_{e}(s(M, t))$, which are weighted by $\alpha$ and $1-\alpha$, respectively. This suggests

$$
u\left(M, t+\tau_{\epsilon}\right)=\alpha u(M+\Delta M, t)+(1-\alpha) u_{e}(s(M, t)),
$$

which, using the Taylor expansion, gives

$$
u_{t}(M, t)=\alpha \frac{\Delta M}{\tau_{\epsilon}} u_{M}(M, t)+(1-\alpha) \frac{u_{e}(s(M, t))-u(M, t)}{\tau_{\epsilon}} .
$$

In general, one could always derive a so-called "anisotropic" acceleration through proper assumptions using the parameters $\Delta M, \tau_{\epsilon}$ and $\alpha$. See [26] and the references therein for the debates on the issue whether traffic flow should be "anisotropic" or "isotropic".

We formulate the anticipation term of Eq. (7) by assuming that $\tau_{\epsilon}$ is proportional to the difference between $s(M+\Delta M, t) \Delta M$ and $s(M, t) \Delta M$, which approximately measure the distance between the particles $M+\Delta M$ and $M+2 \Delta M$, and the distance between the particles $M$ and $M+\Delta M$, respectively. However, $\tau_{\epsilon}$ should be inversely proportional to the difference between the speeds of particles $M+\Delta M$ and $M$, which are approximated by the equilibrium speeds $u_{e}(s(M+\Delta M, t))$ and $u_{e}(s(M, t))$. Accordingly, we assume

$$
\tau_{\epsilon}=\beta \Delta M \frac{s(M+\Delta M, t)-s(M, t)}{u_{e}(s(M+\Delta M, t))-u_{e}(s(M, t))} \doteq \frac{\beta \Delta M}{u_{e}^{\prime}(s(m, t))},
$$

where $\beta>0$ is the proportional coefficient. By replacement, Eq. (7) is rewritten as

$$
u_{t}=\mu u_{e}^{\prime}(s) u_{M}+\frac{u_{e}(s)-u}{\tau},
$$

where $\mu=\alpha \beta^{-1}>0$, and we denote by $(1-\alpha)^{-1} \tau_{\epsilon}=\tau$. We note that neither the weight $\alpha$ nor the coefficient $\beta$ could be taken as constants; at least they should depend on the specific volume $s$ (or the density $\rho$ ), thus we assume $\mu=\mu(s)$. This appears to lead to a complex dependency of the parameter $\tau$.

However, the resultant formulations would demonstrate little difference in solution properties when assuming that $\tau$ is bounded by two positive constants. Therefore, we take $\tau$ as a positive constant for simplicity in the forthcoming studies. In this case, $\tau$ is more commonly referred to as the relaxation time, and Eq. (8) is essentially the Aw-Rascle model [19,20] under the Lagrange coordinate system, which was discussed in $[8,17,18]$. 


\section{Solution Properties of the Continuum Model}

Equations (5) and (8) constitute a complete system for solving the specific volume $s(M, t)$ and the speed $u(M, t)$. The characteristic speeds or eigenvalues of the system are easily indicated as $\lambda_{1}(s, u)=-\mu(s) u_{e}^{\prime}(s)<0$, and $\lambda_{2}(s, u)=0$, by which the propagations are respectively slower than and synchronistic with the motion of particle $M$. This implies that the model is "anisotropic".

\subsection{Linear stability condition}

The system of (5) and (8) has an equilibrium solution $(s, u)=\left(s_{0}, u_{e}\left(s_{0}\right)\right)$, which corresponds to a kinetic speed $-u_{e}^{\prime}\left(s_{0}\right)<0$. The linear stability of this solution requires that the kinetic speed be between the two characteristic speeds [?], which is equivalent to

$$
u_{e}^{\prime}\left(s_{0}\right) \leq \mu\left(s_{0}\right) u_{e}^{\prime}\left(s_{0}\right), \text { or } \mu\left(s_{0}\right) \geq 1 .
$$

It is commonly believed that the equilibrium solution is linearly stable for free and jam density regions, but unstable for a congested density region $[3,11,12]$. Thus, the function $\mu(s)$ is well qualified if $\mu(s)<1$, for $s \in\left(s_{c_{1}}, s_{c_{2}}\right)$, and $\mu(s) \geq 1$, otherwise. Here, $\mu\left(s_{c_{1}}\right)=\mu\left(s_{c_{2}}\right)=1 ; s_{c_{1}}$ and $s_{c_{2}}$ are the critical specific volumes.

\subsection{Traveling wave and solution for a wide moving jam}

Applying the weak solution theory as that discussed in [11-13], we firstly replace $u_{M}$ with $s_{t}$ (see Eq. (5)) in Eq. (8) to derive a conservative equation in the following form:

$$
(u+p(s))_{t}=\frac{u_{e}(s)-u}{\tau},
$$

where $u_{e}(s)$ is assumed to be non-convex, and "pressure" $p(s)$ is related to the first characteristic speed by setting $p^{\prime}(s)=\lambda_{1}(s, u)=-\mu(s) u_{e}^{\prime}(s)$. Then, we briefly indicate a smooth traveling wave profile (transition layer) and a discontinuous traveling wave profile (shock) for the system of (5) and (10) as follows.

By replacing $s=s(N)$ and $u=u(N)$ in Eqs. (5) and (10), where $N=M-q_{0} t$, and $q_{0}<0$ is the traveling wave speed, we easily derive

$$
\frac{d s}{d N}=\frac{u_{e}(s)-u(s)}{\tau q_{0}\left(q_{0}-p^{\prime}(s)\right)} \equiv \phi(s), u(s)=C-q_{0} s,
$$

where $C$ is the integral constant. The second equation in (11) suggests a straight line in the $(s, u)$ phase space. We are interested in a segment solution which intersects with the curve $u=u_{e}(s)$ at $\left(s_{\chi}, u_{\chi}\right)$, for $\chi=A, B, C$. See Fig. 1(a). This implies that

$$
C=q_{0} s_{\chi}+u_{\chi}, u_{\chi}=u_{e}\left(s_{\chi}\right) \text {, for } \chi=A, B, C \text {. }
$$


It is assumed that $s_{C}=s(0)$ and $u_{C}=u(0)$. Then, by Eq. (11) we have

$$
\frac{d N}{d s}=\frac{1}{\phi(s)}, \text { or } N(s)=\int_{s_{C}}^{s} \frac{d s}{\phi(s)} \text {. }
$$

(a)

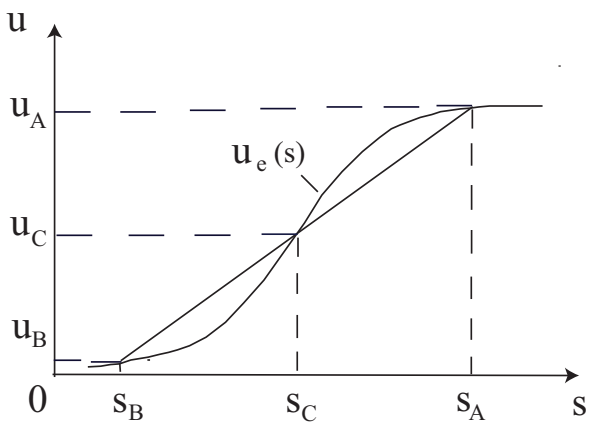

(b)

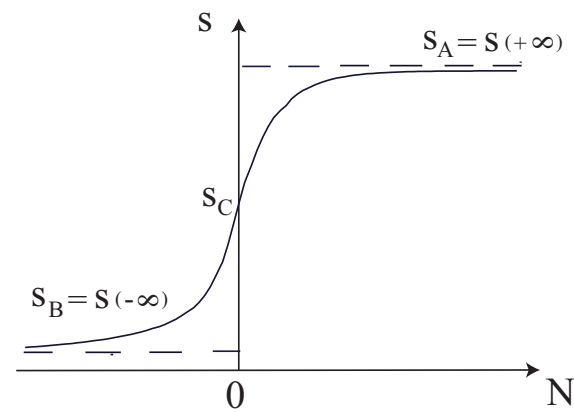

Fig. 1. A traveling wave solution: (a) the phase plot $(s, u)$ compared with the fundamental diagram $u=u_{e}(s)$; and (b) the profile of the transition layer $s=s(N)$.

To ensure the existence of the inverse function $s=s(N)$, we assume the monotonicity of $N(s)$, namely, $\phi(s)$ is always non-negative or non-positive for $s \in\left[s_{B}, s_{A}\right]$. From Fig. 1(a) and Eq. (11), this means that the denominator of $\phi(s)$ changes sign when $s=s_{C}$ with

$$
p^{\prime}\left(s_{C}\right)=q_{0}, \text { and }\left.\frac{d s}{d N}\right|_{s=s_{c}}=\lim _{s \rightarrow s_{C}} \phi(s)=-\frac{u_{e}^{\prime}\left(s_{C}\right)+q_{0}}{\tau q_{0} p^{\prime \prime}\left(s_{C}\right)}<\infty .
$$

It is physically sound that the characteristic speed $p^{\prime}(s)$ should be increasing by $s$ (or decreasing by $\rho=1 / s)$. This implies that $\phi(s)>0$ or $s=s(N)$ is monotonically increasing by $N$. Because

$$
\lim _{s \rightarrow s_{\chi}} \frac{\phi(s)}{s-s_{\chi}}=\frac{u_{e}^{\prime}\left(s_{\chi}\right)+q_{0}}{\tau q_{0}\left(q_{0}-p^{\prime}\left(s_{\chi}\right)\right)} \neq 0, \text { for } \chi=A, B,
$$

we have $N\left(s_{A}\right)=+\infty$, and $N\left(s_{B}\right)=-\infty$, according to the expression of $N(s)$ in Eq. (13). This gives $s_{A}=s(+\infty)$, and $s_{B}=s(-\infty)$. The profile of the traveling wave solution $s=s(N)$ is illustrated in Fig. 1(b), which corresponds to (and thus is also called) a transition layer in the literature [3].

(a)

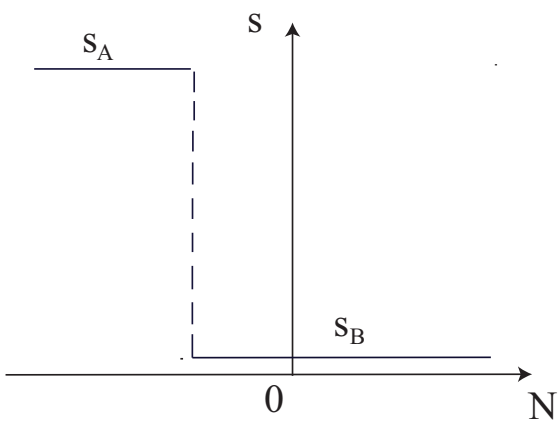

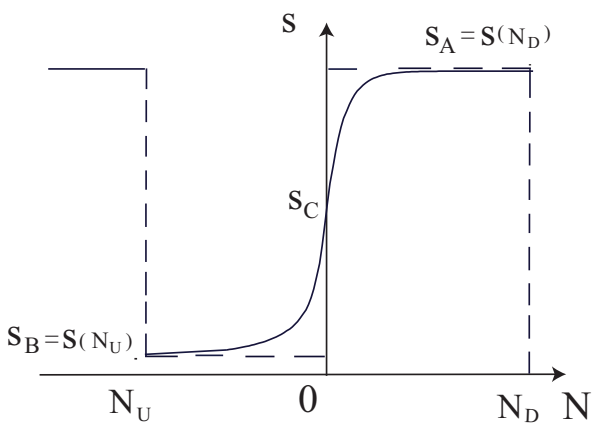

Fig. 2. (a) the profile of a shock; and (b) the profile of the wide moving jam that is composed of a shock and a transition layer. 
A discontinuous traveling wave or shock that connects two equilibrium states (Fig. 2(a)) is determined by applying the Rankine-Hugoniot conditions to the system of (5) and (10), which read:

$$
\begin{gathered}
q_{0}\left(s_{A}-s_{B}\right)=-\left(u_{e}\left(s_{A}\right)-u_{e}\left(s_{B}\right)\right), \\
u_{e}\left(s_{A}\right)+p\left(s_{A}\right)=u_{e}\left(s_{B}\right)+p\left(s_{B}\right) .
\end{gathered}
$$

Here, the two constant equilibria are also denoted by $\left(s_{A}, u_{e}\left(s_{A}\right)\right)$ and $\left(s_{B}, u_{e}\left(s_{B}\right)\right)$, which are respectively on the left and right hand sides of the shock; the shock speed is also denoted by $q_{0}$. We note that the shock is associated with the first characteristic field; it is physically valid only when $\lambda_{1}\left(s_{A}, u_{e}\left(s_{A}\right)\right)>\lambda_{1}\left(s_{B}, u_{e}\left(s_{B}\right)\right)$, or $s_{A}>s_{B}$, according to the Lax-entropy condition [27].

Solution of a wide moving jam is composed of a shock (as the upstream front) and a transition layer (as the downstream front), with the latter profile being well approximated by the two equilibrium constant states $\left(s_{B}, u_{e}\left(s_{B}\right)\right)$ and $\left(s_{A}, u_{e}\left(s_{A}\right)\right)$ respectively, in $s \in\left(-\infty, N_{U}\right]$ and $s \in\left[N_{D},+\infty\right)$ for some $N_{U}$ and $N_{D}$ (Fig. 2(b)). For this solution, all parameters using the same denotations in Eqs. (12)-(16) can be truly set as the same, and Eqs. (12), (14), (15) and (16) imply four independent algebraic equations, by which the four characteristic parameters $q_{0}, s_{A}, s_{B}$ and $s_{C}$ could be uniquely solved.

\section{Semi-Discrete Model and Numerical Results}

It is convenient to describe the motion of the particles under the Lagrange coordinate system. One way is to numerically solve the partial differential conservation system of (5) and (10), and then to derive $x(M, t)$ through Eqs. (3)-(4). However, it is intuitive and straightforward to discretize Eq. (3) by

$$
s_{m}(t)=\frac{x_{m+1}(t)-x_{m}(t)}{\Delta M}
$$

and then rewrite Eq. (4) as

$$
\frac{d x_{m}(t)}{d t}=u_{m}(t)
$$

Here and hereafter, we denote a solution variable $A$ by $A_{m}(t)=A(M, t)$, and $A_{m+1}(t)=A(M+$ $\Delta M, t)$. Because the set of particles in discretization is enumerable for any fixed increment $\Delta M, m$ is taken as an integer. Using Eq. (17), Eq. (10) is rewritten as

$$
\frac{d}{d t}\left[u_{m}(t)+p\left(\frac{x_{m+1}(t)-x_{m}(t)}{\Delta M}\right)\right]=\frac{1}{\tau}\left[u_{e}\left(\frac{x_{m+1}(t)-x_{m}(t)}{\Delta M}\right)-u_{m}(t)\right] .
$$

Equations (18)-(19) constitute a complete semi-discrete system for solution. Because it can be verified that each equation of (3)-(5) is implied by the two others, the semi-discrete system can be shown to be consistent with the continuum system of (5) and (10). This means that the former system will 
become the latter system for $\Delta M \rightarrow 0$, provided that the term on the right hand side of Eq. (17) converges.

Recall that $\Delta M$ is the mass of the fluid between particles $m$ and $m+1$. Then, the position $x_{m}(t)$ and the increment $\Delta M$ are related to the specific volume $s(x, t)$ or density $\rho(x, t)=(s(x, t))^{-1}$ through the formula:

$$
\Delta M=\int_{x_{m}(t)}^{x_{m+1}(t)}(s(x, t))^{-1} d x=\int_{x_{m}(t)}^{x_{m+1}(t)} \rho(x, t) d x
$$

\subsection{Linear stability of the equilibrium solution}

Assume that $\left(x_{m}^{0}(t), u_{m}^{0}(t)\right)$ is an equilibrium solution of system (18)-(19). We derive $x_{m+1}^{0}(t)-$ $x_{m}^{0}(t)=s_{0} \Delta M$ by setting $s(x, t)=s_{0}$ in Eq. (20), where $s_{0}$ is constant. Then, we substitute this into Eq. (19) to derive an unique solution $u_{m}^{0}(t)=u_{e}\left(s_{0}\right)$, which in turn gives $x_{m}^{0}(t)=u_{e}\left(s_{0}\right) t+$ $m \Delta M s_{0}+C$, where $C$ is an arbitrary constant. This solution corresponds to the equilibrium solution $\left(s_{0}, u_{e}\left(s_{0}\right)\right)$ of system (5) and (10) (see the discussion in Section 3.1).

To examine the linear stability of the aforementioned equilibrium solution, we assume a perturbed solution: $x_{m}(t)=x_{m}^{0}(t)+y_{m}(t), u_{m}(t)=x_{m}^{\prime}(t)=u_{e}\left(s_{0}\right)+y_{m}^{\prime}(t)$, where $y_{m}(t)$ is the perturbation. Substitute the perturbed solution into Eq. (19) and apply the Taylor expansion to the functions $p^{\prime}(s)$ and $u_{e}(s)$ at $s=s_{0}$ in the resultant equation. Then, by omitting the nonlinear terms, we derive the following linear ODE equation:

$$
y_{m}^{\prime \prime}=\frac{1}{\tau}\left[\frac{u_{e}^{\prime}\left(s_{0}\right)}{\Delta M}\left(y_{m+1}(t)-y_{m}(t)\right)-y_{m}^{\prime}\right]-\frac{p^{\prime}\left(s_{0}\right)}{\Delta M}\left(y_{m+1}^{\prime}-y_{m}^{\prime}\right) .
$$

By substituting $y_{m}(t)$ with the Fourier mode $\exp \left(i k m \Delta M-i k \sigma_{k} t\right)$ in Eq. (21), where $m \Delta M$ represents the Lagrange coordinate $M, \sigma_{k}=\sigma_{1}+\sigma_{2}(i k)$, and $\sigma_{1}$ is the wave speed of the small perturbation, we derive a polynomial equation of $k$. In this case, we assume a small wave number $k \ll 1$ and thus leave only two lower order terms in the polynomial equation, which is balanced by

$$
\sigma_{1}=-u_{e}^{\prime}\left(s_{0}\right), \quad \sigma_{2}=\Delta M\left(u_{e}^{\prime}\left(s_{0}\right)+p^{\prime}\left(s_{0}\right)\right)-\frac{\Delta M^{2}}{2 \tau} .
$$

For stability, the amplitude $\exp \left(k^{2} \sigma_{2}\right)$ of the perturbation $y_{m}(t)=\exp \left(i k m \Delta M-i k \sigma_{k} t\right)$ should not be greater than 1 , or $\sigma_{2}$ should be non-positive. This suggests the following linear stability condition of the equilibrium solution $\left(x_{m}^{0}(t), u_{m}^{0}(t)\right)$ :

$$
u_{e}^{\prime}\left(s_{0}\right)+p^{\prime}\left(s_{0}\right) \leq \frac{\Delta M}{2 \tau} .
$$

We note that Eq. (22) is consistent with that of Eq. (9). Precisely, Eq. (22) reduces to Eq. (9) as $\Delta M \rightarrow 0$, where $\mu\left(s_{0}\right)=-p^{\prime}\left(s_{0}\right) / u_{e}^{\prime}\left(s_{0}\right)$. Also compare this discussion for consistency with that below Eq. (19). 


\subsection{A resultant car-following model}

Let $\Delta M=1$, then Eq. (20) implies that the mass between two adjacent particles is unit. In this case, the semi-discrete model is directly related to traffic flow in that all particles can be viewed as cars and Eq. (20) actually implies that there is just one car between the heads of two adjacent cars. Actually, the system of (18) and (19) reduces to a car-following model as follows:

$$
\begin{gathered}
\frac{d x_{m}(t)}{d t}=u_{m}(t), \\
\frac{d}{d t}\left[u_{m}(t)+p\left(x_{m+1}(t)-x_{m}(t)\right)\right]=\frac{1}{\tau}\left[u_{e}\left(x_{m+1}(t)-x_{m}(t)\right)-u_{m}(t)\right],
\end{gathered}
$$

which is exactly the same as that discussed in [8]. Moreover, the specific volume $s_{m}=x_{m+1}(t)-x_{m}(t)$ turns out to be the headway, and an approximation of Eq. (20) retrieves the relation:

$$
s_{m}=\frac{1}{\rho},
$$

which is well-known in the literature.

\subsection{Comparison between numerical and analytical solutions}

The foregoing discussion suggests a substantial relation between the semi-discrete model (Eqs. (18)(19)) and the continuum model (Eqs. (5) and (10)). Recall the discussions below Eqs. (19) and (22) for consistency. The semi-discrete model should similarly suggest a solution for wide moving jams. Moreover, the characteristic parameters of this solution should converge to those that are analytically derived in Section 3.2, which is based on the continuum model. In the following, we indicate this convergence through proper calibration of the model parameters.

By setting $\Delta M=1$ and $s(x, t)$ (or $\rho(x, t)$ ) to be constant in Eq. (20), we have $s(x, t)=$ $x_{m+1}(t)-x_{m}(t)$. In this case, particle $M$ represents a car in traffic labeled by the integer $m$; $s(x, t)$ is actually the headway (see Eqs. (23)-(25)), which cannot be smaller than the car length $l$. Therefore, $l$ is taken as a characteristic parameter in general such that $s \geq l$, or $\rho \leq \rho_{j a m}$, where $\rho_{j a m} \equiv l^{-1}$ denotes the maximal density. Referring to [8] and [13], we respectively define the fundamental diagram and the "pressure" as follows:

$$
u_{e}(s)=u_{f} \frac{\tanh (s / l-r)+\tanh (r-1)}{1+\tanh (r-1)}, \quad p(s)=\alpha u_{f}(l / s)^{\gamma},
$$

where $u_{f}=30 \mathrm{~m} / \mathrm{s}, r=3, l=4.5 \mathrm{~m}, \alpha=2.5$, and $\gamma=0.5$. By these parameters and Eq. (9), the critical specific volumes are determined as $s_{c_{1}}=10.7170 \mathrm{~m}$, and $s_{c_{2}}=18.7949 \mathrm{~m}$. Moreover, the initial conditions of the semi-discrete model and the continuum model are related through

$$
\Delta M=\int_{x_{m}(0)}^{x_{m+1}(0)} \rho(x, 0) d x, u_{m}(0)=u\left(x_{m}(0), 0\right),
$$


where $\rho(x, 0)=(s(x, 0))^{-1}$ (see also Eq. (20)). Given $\Delta M$ and defined by $x_{1}(0)=0$, the initial positions $x_{m}(0)$ of all particles can be (numerically) determined by the first equation in (26), and thus all $u_{m}(0)$ are determined by the second equation.

For numerical simulation of the semi-discrete model, we take a ring road $[0, L]$ such that the position of $x_{m}(t)($ for $t \neq 0)$ is reset as $x_{m}(t)-L$ if $x_{m}(t)>L$. Initially, the total number of particles in the ring is identified as $n$, if $x_{n}(0) \leq L$ and $x_{n+1}(0)>L$. This usually gives rise to a small error, but this vanishes as $\Delta M \rightarrow 0$. For the transformation of (26), the initial conditions are given by

$$
\rho(x, 0)=\rho_{0}+\delta \rho_{0} \sin \left(\frac{2 \pi x}{L}\right), u(x, 0)=10.5 \mathrm{~m} / \mathrm{s},
$$

which suggests a global perturbation to the equilibrium solution $\left(s_{0}, u_{e}\left(s_{0}\right)\right)\left(s_{0}=\rho_{0}^{-1}\right)$. Here, $\rho_{0}$ can be easily indicated as the integral average in the ring.

To derive the profile of a wide moving jam that is well approximated by the description in Section $3.2, s_{0}$ should be close to the middle of the unstable interval $\left(s_{c_{1}}, s_{c_{2}}\right)$, and we set $s_{0}=13.5 \mathrm{~m}$ for the simulation. Here, the physics is simply that, with a sufficiently large amount of mass in the ring, the perturbed unstable equilibrium state $\left(s_{0}, u_{e}\left(s_{0}\right)\right)$ would inevitably evolve into a stable wide moving jam. We note that the moving jam will become narrower if $s_{0}$ increases, and that one would more likely derive an "anti moving jam" that is comparable to an anti-cluster discussed in Kerner [3] if $s_{0}$ decreases. Moreover, the length of the "anti moving jam" decreases with the decrease in $s_{0}$. See also discussions in $[7,11-13]$.

Table 1 Comparison between the numerical solution of the semi-discrete model and the analytical solution of the continuum model. With the refinement of $\Delta M$, the characteristic parameters in the former solution converge to those solved from Eqs. (12), (14), (15) and (16), which correspond to $\Delta M=0$.

\begin{tabular}{ccccc}
\hline$\Delta M$ & $s_{B}$ & $s_{A}$ & error in $s_{A}$ & convergence order in $s_{A}$ \\
\hline 1 & 6.7056 & 21.6064 & 0.9536 & \\
$1 / 3$ & 6.5832 & 22.1982 & 0.3618 & 0.8822 \\
$1 / 9$ & 6.5554 & 22.4495 & 0.1105 & 1.0796 \\
$1 / 27$ & 6.5496 & 22.5150 & 0.0450 & 0.8177 \\
$1 / 81$ & 6.5474 & 22.5469 & 0.0131 & 1.1233 \\
0 & 6.5465 & 22.5600 & & \\
\hline
\end{tabular}

We simply adopt the first-order Euler forward difference scheme for the time discretization of system (18)-(19). With refinement of the increment $\Delta M$, the characteristic parameters (the minimal and maximal specific volumes $s_{B}$ and $s_{A}$ ) are shown in Table 1, which approximately suggests a first order convergence to those derived by solving the algebraic equations of (12), (14), (15) and (16). 
This agrees with a truncation error of $O(\Delta M)$ between Eqs. (10) and (19), which can be easily observed through a Taylor expansion in Eq. (19).

The profiles and phase plots of the numerical solutions for $\Delta M=1$ and $\Delta M=1 / 81$ are shown in Fig. 3. Compare Fig. 3 with Figs. 1 and 2. The profile of the numerical solution (Fig. 3(a) versus Fig. 2(b)) clearly includes a transition layer and a shock, which respectively constitute the upstream and downstream fronts of the wide moving jam. Moreover, the phase plot of the numerical solution (Fig. 3(b) versus Fig. 1(a)) is almost a straight line, which suggests that the solution is very close to a travel wave. We note that the fold lines in Fig. 3(b) represent a smoothed shock path due to the numerical viscosity. Combined with Table 1, Fig. 3 visualizes the comparison between the solutions of the continuum model (represented by $\Delta M=1 / 81$ ) and the corresponding car-following model (represented by $\Delta M=1$ ). This also shows that the analytical solution of the former model is an adequate approximation of the latter.
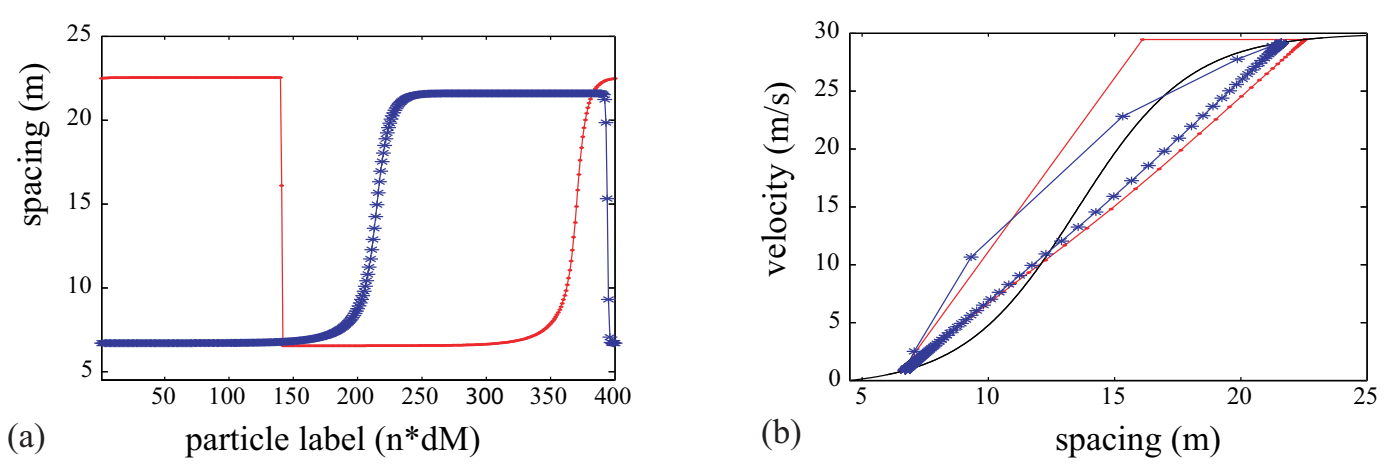

Fig. 3. Solution of a wide moving jam developed from a perturbed unstable equilibrium state, by solving the semi-discrete model with $\Delta M=1$ (blue and starred) and $\Delta M=1 / 81$ (red and dotted). (a) the solution profile $\left((m-1) \Delta M, \Delta x_{m} / \Delta M\right)$, where $\Delta x_{m}=x_{m+1}-x_{m}$, and $m=1, \ldots, n ;\left(\right.$ b) The phase plot $\left(\Delta x_{m} / \Delta M, u_{m}\right)$ compared with the fundamental diagram $u=u_{e}(s)$.

\section{Conclusions}

The basic concept of the Lagrange coordinates is exploited to establish the relationship between the continuum model and the semi-discrete model. This provides a platform to study the characteristic features of wide moving jams in both models, which is very important for the further development of Kerner's three-phase traffic theory.

The consistency between the two models more generally suggests that the theories for these two types of models could be developed in parallel. On the one hand, the shortcomings of the existing (semi-discrete) car-following models could be overcome by establishing similar correlations and then by adopting the theoretical results (e.g., those in $[8,9,12,12,13,22]$ ) generated in the study of the 
continuum model. On the other hand, theoretical findings or simulations that significantly reflect the characteristic features of real traffic in existing car following models could be incorporated into the continuum models for development through the correlation.

\section{Acknowledgements}

The study was jointly supported by grants from the National Natural Science Foundation of China (11072141), and the University Research Committee and HKU SPACE Research Fund (201007176059), and an Outstanding Researcher Award from the University of Hong Kong, and Shanghai Program for Innovative Research Team in Universities.

\section{References}

[1] B.S. Kerner, The Physics of Traffic, Springer, Berlin, New York, (2004).

[2] B.S. Kerner, Introduction of Modern Traffic Flow Theory and Control, Springer, Berlin, New York, (2009).

[3] B. S. Kerner and P. Konhauser, Structure and parameters of clusters in traffic flow, Phys. Rev. E 50 (1994), 54-83.

[4] H. J. Payne, Models of freeway traffic and control, In: A.G. Bekey (ed.) Mathematical Models of Public Systems, Simulation Council Proc., La Jola, 1(1971), 51-61.

[5] K. Gao, R. Jiang, S.X. Hu, B.H. Wang, Q.S. Wu, Cellular-automaton model with velocity adaptation in the framework of Kerner's three-phase traffic theory, Physical Review E 76(2) (2007), 026105.

[6] B. Jia, X.G. Li, T. Chen, R. Jiang and Z.Y. Gao, Cellular automaton model with time gap dependent randomization under Kerners three-phase traffic theory, Transportmetrica 7(2) (2011), 127-140.

[7] B.S. Kerner, S.L. Klenov, and P. Konhäuser, Asymptotic theory of traffic jams. Physical Review E 56 (1997), 4200-4216.

[8] J.M. Greenberg, Congestion redux, SIAM J. Appl. Math. 64(4) (2004), 1175-1185.

[9] F. Siebel and W. Mauser, On the fundamental diagram of traffic flow, SIAM J. Appl. Math. 66 (2006), 1150-1162.

[10] F. Siebel and W. Mauser, Synchronized flow and wide moving jams from balanced vehicular traffic, Phys. Rev. E 73 (2006), 066108. 
[11] P. Zhang, S. C. Wong and S. Q. Dai, Characteristic parameters of a wide cluster in a higher-order traffic flow model, Chinese Physics Letters 23 (2006), 516-519.

[12] P. Zhang and S.C. Wong, Essence of conservation forms in the traveling wave solutions of Higher-Order Traffic Flow Model, Phys. Rev. E 74 (2006), 026109.

[13] R.Y. Xu, P. Zhang, S.Q. Dai and S.C. Wong, Admissiblity of a wide cluster solution in "anisotropic" higher-order traffic flow models, SIAM J. Appl. Math. 68 (2007), 562-573.

[14] M. Bando, K. Hasebe, A. Nakayama, A. Shibata, and Y. Sugiyama, Dynamical model of traffic congestion and numerical simulation. Phys. Rev. E 51 (1995), 1035-1042.

[15] T. Nagatani, Soliton and kink jams in traffic flow with open boundaries, Phys. Rev. E 60 (1999), 180-187.

[16] D. Helbing and B. Tilch. Generalized force model of traffic dynamics. Phys. Rev. E 58 (1998), 133-138.

[17] A. Aw, A. Klar, T. Materne, and M. Rascle, Derivation of continuum traffic flow models from microscopic follow-the-leader models, SIAM J. Appl. Math. 63 (2002), 259-278.

[18] J. Greenberg, Extensions and amplifications of a traffic model of Aw and Rascle, SIAM J. Appl. Math. 62 (2001), 729-745.

[19] A. Aw and M. Rascle, Resurrection of "second order" models of traffic flow, SIAM J. Appl. Math. 60 (2000), 916-938.

[20] M. Rascle, An improved macroscopic model of traffic flow: derivation and links with the LighthillWhitham model, Mathematical and Computer Modelling 35 (5/6) (2002), 581-590.

[21] H.M. Zhang,A non-equilibrium traffic model devoid of gas-like behavior, Transp. Res. B 36 (2002), 275-290.

[22] P. Zhang, S.C. Wong and S.Q. Dai, A conserved higher-order anisotropic traffic flow model: description of equilibrium and non-equilibrium flows, Transpn. Res. 43B (2009), 562-574.

[23] Newell, G.F.,A simplified theory of kinematic waves in highway traffic. I: General theory. II: Queuing at freeway bottlenecks. III: Multi-destination flows. Transpn. Res. 27B (1993), 281-287.

[24] C.F. Daganzo, A variational formulation of kinematic waves: basic theory and complex boundary conditions, Transpn. Res. 39B (2005), 187-196.

[25] T.Q. Tang, H.J. Huang and H.Y. Shang, A new macro model for traffic flow with the consideration of the driver's forecast effect, Physics Letters A 374 (2010), 1668-1672.

[26] H.M. Zhang, Comment on "On the controversy around Daganzo's requiem for and Aw-Rascle's resurrection of second- order traffic flow models" by D. Helbing and A.F. Johansson, Eur. Phys. J. B 69 (2009), 563-568. 
[27] P.D. Lax, Shock waves and entropy, In: E.H. Zarantonello (ed.) Contributions to Nonlinear Functional Analysis, Academic Press, New York, (1971). 